\title{
Colorectal adenocarcinoma with enteroblastic differentiation: diagnostic challenges of a rare case encountered in clinical practice
}

\author{
Evi Abada ${ }^{1}$, Ifeoma C. Anaya ${ }^{2}$, Othuke Abada ${ }^{3}$, Anthony Lebbos ${ }^{4}$, Rafic Beydoun $^{1}$ \\ 'Department of Pathology, Wayne State University School of Medicine/Detroit Medical Center, Detroit, Ml; '2Larkin Community Hospital, South Miami, FL; \\ ${ }^{3}$ Ascension St. John Hospital, Detroit, Ml; ${ }^{4}$ Michigan State University, East Lansing, Ml, USA
}

Colorectal adenocarcinoma with enteroblastic differentiation (CAED) is a rare subtype of colonic adenocarcinoma characterized by increased $\alpha$-fetoprotein (AFP) production and the expression of at least one enteroblastic marker including AFP, glypican 3 (GPC3), or Spalt like transcription factor 4 (SALL4). We report a case of a 26-year-old female who presented with low back pain and constipation which persisted despite supportive measures. Imaging revealed multiple liver lesions and enlarged retroperitoneal nodes. Tumor markers including AFP were markedly elevated. On biopsy, samples from the liver revealed infiltrating glands lined by columnar-type epithelium with mostly eosinophilic granular to focally clear cytoplasm. By immunohistochemistry, the tumor showed immunoreactivity with AFP, hepatocyte antigen, GPC3, SALL4, CDX2, SATB2, and cytokeratin 20. A colonoscopy performed subsequently revealed a mass in the sigmoid colon and biopsy of this mass revealed a similar histology as that seen in the liver. A diagnosis of CAED was made, following the results of gene expression profiling by the tumor with next-generation sequencing which identified pathogenic variants in MUTYH, TP53, and KDM6A genes and therefore supported its colonic origin. Cases such as this underscores the use of ancillary diagnostic techniques in arriving at the correct diagnosis in lesions with overlapping clinicopathologic characteristics.

Key Words: Colorectal neoplasms; Colorectal adenocarcinoma with enteroblastic differentiation; Alpha fetoprotein

Received: August 21, 2021 Revised: October 26, 2021 Accepted: October 27, 2021

Corresponding Author: Evi Abada, MD, MS, Department of Pathology, Wayne State University School of Medicine/Detroit Medical Center, 3990 John R. Road, Detroit, Ml 48201, USA

Tel: +1-313-577-1102, Fax: +1-313-577-0057, E-mail: gs5839@wayne.edu

Colorectal adenocarcinoma with enteroblastic differentiation (CAED) is a rare subtype of colorectal adenocarcinoma with expression of enteroblastic markers including glypican 3 (GPC3), Spalt like transcription factor 4 (SALL4), and $\alpha$-fetoprotein (AFP) [1]. The incidence of CAED compared with other types of colorectal carcinoma (CRC) have been reported to be $0.72 \%$ [1]. Many cases consist of cells which have a clear cytoplasm, but some cases present with eosinophilic cytoplasm, while others may be poorly differentiated. The unifying characteristic for these different phenotypes is the expression of at least one enteroblastic marker by the tumor.

In contrast to the conventional type, gastrointestinal adenocarcinoma with enteroblastic differentiation tends to be aggressive with frequent lymphovascular infiltration, metastasis to the liver, and lymphatics. This corresponds with a poor prognosis [2,3]. Most AFP-producing tumors are reported to arise from organs of the foregut endoderm such as the stomach, bile duct, and pancreas [4]. However, AFP-producing colorectal adenocarcinoma is extremely rare, possibly because the colorectum originates from the hindgut endoderm [4,5].

AFP is an oncofetal glycoprotein produced by the fetal liver, yolk sac, and a small amount from the fetal gastrointestinal epithelium [6]. Serum levels are elevated after birth but decrease significantly by the second year of life [4]. Thus, an increase in serum AFP is abnormal in adults as it is produced by certain tumors, making it a useful tumor marker for the diagnosis and monitoring of treatment [7].

In addition to AFP, the oncofetal proteins GPC3 and SALL4, expressed in germ cell tumors, are immunohistochemically associated with AFP-producing gastric cancers and their related counterparts. Other markers include cytokeratin (CK) 7, CK 20, and caudal-type homeobox 2 (CDX2) which aid in differentiating between tumors of the breast, gynecological tract, liver, and lung origin [8]. 
Here we present a case of chemo-refractory CAED in a young female who initially presented with multiple liver lesions, and a mass in the sigmoid colon, with diagnostic considerations of a primary hepatocellular carcinoma (HCC), germ cell tumor, and CAED, and the use of ancillary diagnostic techniques in arriving at the correct diagnosis.

\section{CASE REPORT}

This is a case of a 26-year-old female who presented clinically with a history of low backache and constipation, which persisted despite supportive measures. Imaging studies revealed multiple liver lesions, with enlarged retroperitoneal lymph nodes. Tumor markers at presentation included an AFP of 39,493 ng/mL, car-

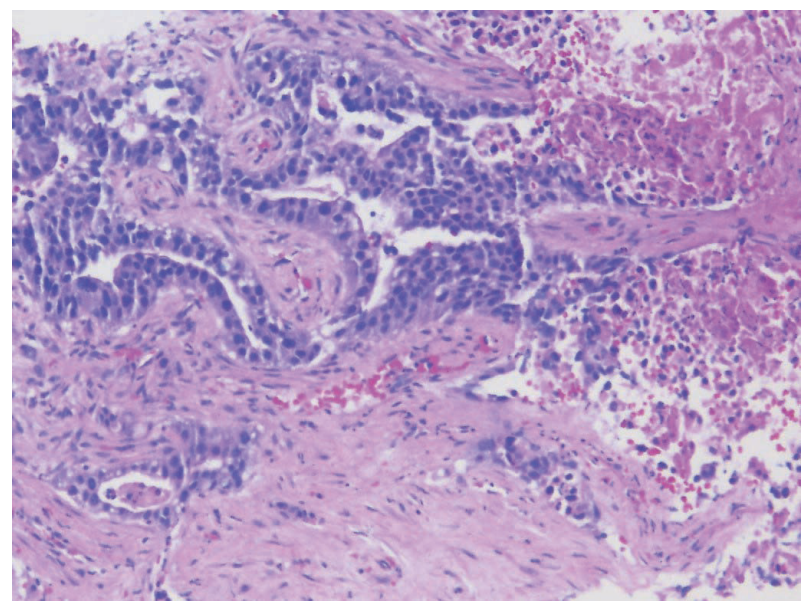

Fig. 1. Liver mass biopsy showing infiltrative malignant glands and a focus of necrosis is also seen. cinoembryonic antigen of 3,040 $\mathrm{ng} / \mathrm{mL}$, cancer antigen (CA) 19-9 of $621 \mathrm{U} / \mathrm{mL}$, and CA 125 of $42 \mathrm{U} / \mathrm{mL}$.

A biopsy of her liver nodules was performed and histopathologic review of tissue sections revealed segmental necrosis with infiltrating adenocarcinoma (Fig. 1). The tumor cells had moderate amounts of mostly granular eosinophilic to focally clear cytoplasm (Fig. 2A) and nuclei with densely homogenous chromatin and occasional inconspicuous nucleoli (Fig. 2B). Immunohistochemistry (IHC) revealed that the tumor cells were immunoreactive for AFP (Fig. 3A), Sal-like protein 4 (Fig. 3B), CK20 (Fig. 3C), and showed strong diffuse immunoreactivity with CDX2 (Fig. 3D). Hepatocyte specific antigen was also positive on IHC. However, CK7, PAX8, and S100 were all negative. A colonoscopy performed subsequently, revealed a mass in the sigmoid colon and biopsy of this mass revealed a similar histology as that seen in the liver (Fig. 4A-C). IHC expression in the colonic tumor showed similar expression as that in the liver. Considering the fact that CDX2 positivity would be unlikely in a primary HCC, a diagnosis of CAED was favored based on a combination of serum tumor markers, tumor morphology, and IHC expression in the tumor cells. Additional testing performed on the tumor cells include human epidermal growth factor receptor 2 , which was interpreted as equivocal $(2+)$ on IHC and negative by fluorescence in situ hybridization. KRAS mutations were identified in the tumor cells, but programmed death-ligand 1 (PD-L1) was negative. IHC stains for mismatch repair (MMR) proteins MLH1, MSH2, MSH6, and PMS2 showed no loss of expression (no evidence of MMR deficiency). She was commenced on targeted therapy including FOLFOX (leucovorin calcium [folinic
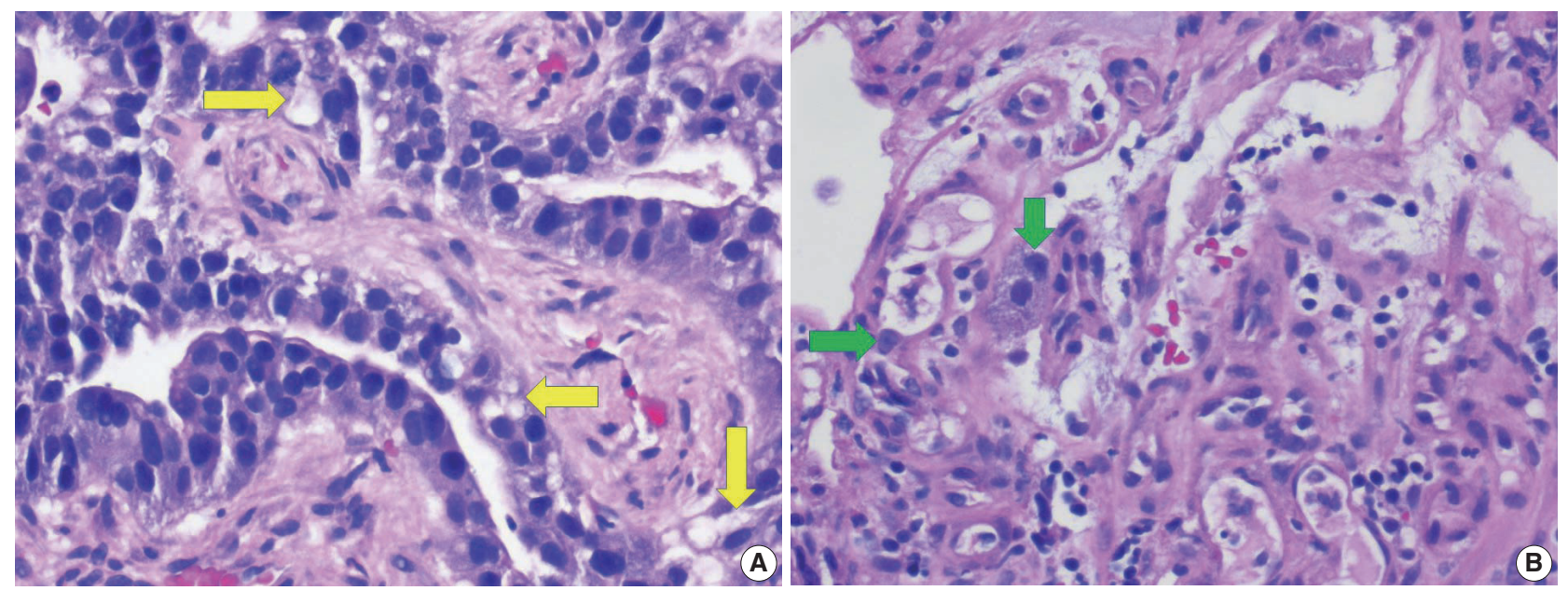

Fig. 2. (A) Higher magnification of hepatic mass showing cells with moderate amounts of mostly granular eosinophilic to focally clear cytoplasm (arrows). (B) Higher magnification of hepatic mass showing tumor cells with moderate amounts of mostly granular eosinophilic cytoplasm and inconspicuous nucleoli (arrows). 
acid], fluorouracil, and oxaliplatin) and bevacizumab. However, with suboptimal improvement in her clinical disease, a second opinion was sought.

She underwent additional imaging studies which revealed new satellite lesions in the liver and previously undiscernible lesions in bilateral ovaries, which was highly suspicious for advancing metastatic disease. With the discovery of new lesions in the ovary, her previous biopsies from the liver nodules and colonic mass were re-reviewed and a diagnosis of poorly differentiated carcinoma, most suggestive of yolk sac tumor was rendered in the original
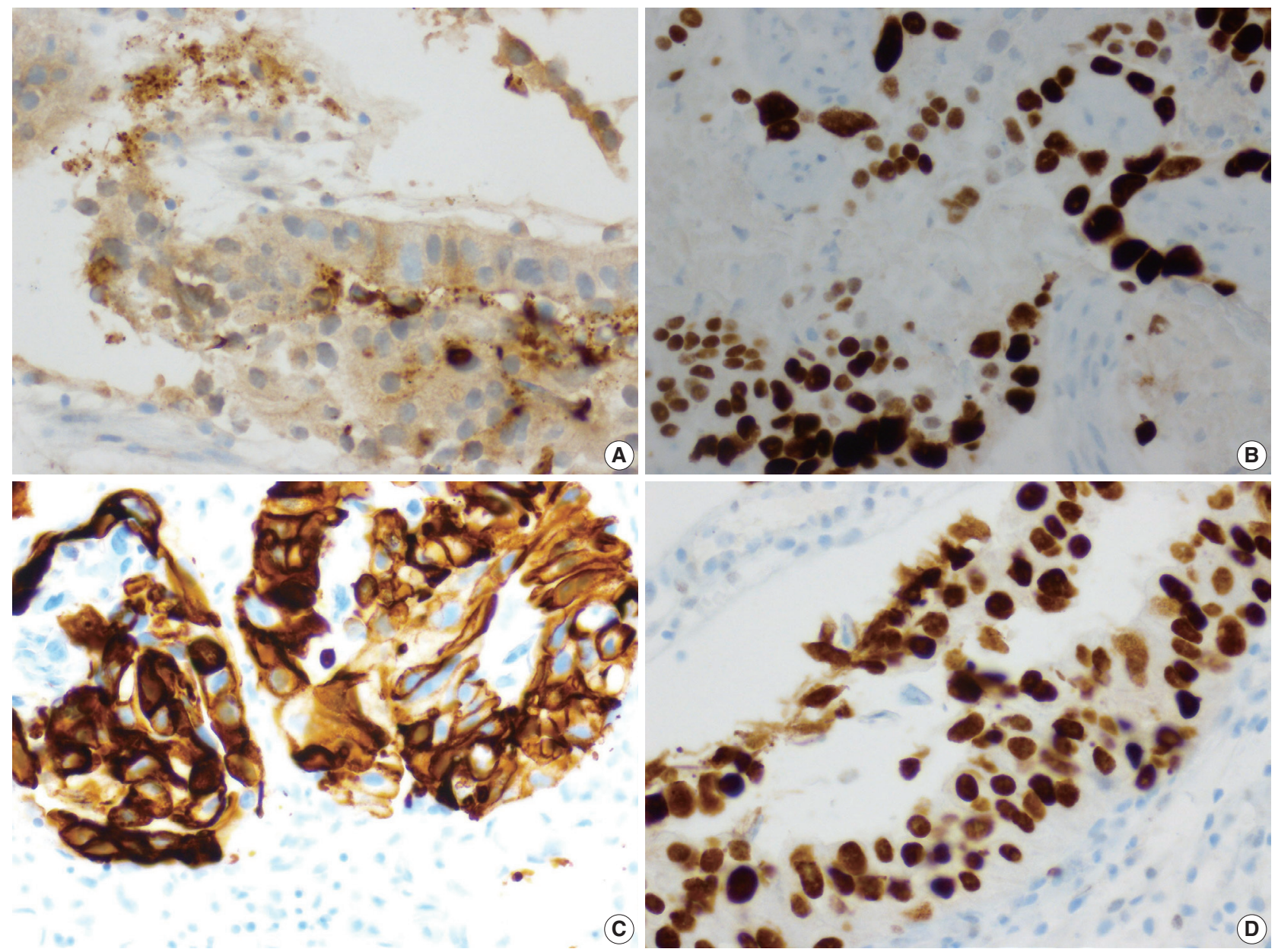

Fig. 3. Immunohistochemical stains show that the tumor cells are immunoreactive for $\alpha$-fetoprotein (A), Sal-like protein 4 (B), cytokeratin 20 (C), and CDX2 (D).
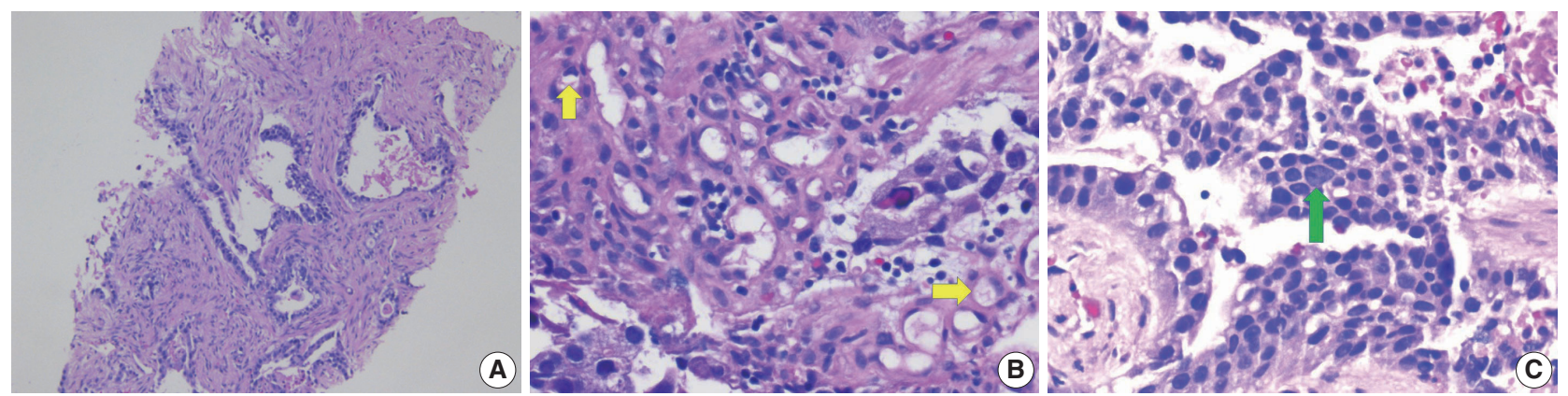

Fig. 4. (A) Biopsy of sigmoid colon mass showing infiltrative malignant glands. (B) Higher magnification of colonic mass showing cells with moderate amounts of mostly granular eosinophilic to focally clear cytoplasm (arrows). (C) Higher magnification of colonic mass showing tumor cells with moderate amounts of mostly granular eosinophilic cytoplasm and a cell with inconspicuous nucleolus (arrow). 
liver and colonic tumor specimens. Other differentials that were considered included a mixed germ cell tumor or teratoma. She was commenced on chemotherapy for germ cell tumors (cisplatin, etoposide, and bleomycin) but showed disease progression on imaging studies. This prompted a decision to send the specimens from the liver and colon for tumor gene expression profiling by next-generation sequencing (NGS). NGS identified several pathogenic variants including MUTYH (exon 13, p.G396D), TP53 (exon 5, p.R175H), and KDM6A (exon 16, p.S532fs). Additionally, estrogen receptor and progesterone receptor by IHC were both negative. Based on the constellation of clinical findings and test results, a diagnosis of CAED was upheld and she is being evaluated for autologous transplant for chemo-refractory CAED.

\section{DISCUSSION}

The incidence of AFP-producing gastric carcinoma has been documented to be within the range of 1.3\%-15\% worldwide with the incidence of the colorectal variant being significantly lower compared to the gastric [9]. This was first reported by Nakajima et al. in 1985 [10]. Prior reports suggest that CAED is more common in males and tends to be located in the left colon $[1,2]$.

Furuya et al. in their case report in 2011 reported the first case of primary AFP-producing clear cell adenocarcinoma of the colon in an 81-year-old man, which is considered extremely rare when compared to that of gastric origin [11]. They made a distinction between a primary colonic tumor and metastasis from the gynecological tract or other organs by whole-body computed tomography $(\mathrm{CT})$ and immunostaining. CT results revealed tumor deposits in the colon and lung only with $\mathrm{CK} 7$ negative and CD20 positive immunoreactivity [11].

Histologically, the tumor cells display a spectrum of morphologic patterns. One report describes tumor cells that displayed typical adenocarcinoma morphologies composed of cuboidal or columnar cells with clear cytoplasm resembling the primitive gut [12]. The enteroblastic component was seen occurring concurrently with typical well or moderately differentiated tubular adenocarcinomas [12]. Another report describes the tumor's morphology as characterized by a solid-type poorly differentiated carcinoma, mainly composed of eosinophilic cells with focal glandular differentiation on hematoxylin and eosin stains [13].

Our patient is a young, 26-year-old female, an interesting demographic in contrast to most documented cases of CAED. Her primary tumor was located on the left side (sigmoid colon) with multiple liver, nodal, and bilateral ovarian involvements war- ranting the diagnostic consideration of mixed germ cell tumor especially with unresponsiveness to conventional chemotherapy. However, with tumor progression on imaging following the administration of chemotherapy for germ cell tumor and the IHC profile, a diagnosis of chemo-refractory CAED was ultimately favored, based on the constellation of clinicopathologic findings and results of gene expression of the tumor cells by NGS. Of note is that the MUTYH gene encodes a DNA glycosylase involved in oxidative DNA damage repair and mutations in the gene predispose to colon and stomach cancers. A germline mutation in TP53 predisposes to multiple cancers, including colorectal cancer.

Clear cell changes were proposed by Eloy et al. [14] to be a result of degenerative changes with an accumulation of cytoplasmic lipid-like material and not necessarily the accumulation of cytoplasmic glycogen and mucin. Therefore, mucin and glycogen may go undetected in some cases of CAED [14]. In our case, we observed mostly eosinophilic granular cytoplasm, with only a focal clear cell component (Fig. 2).

AFP production in gastric carcinoma was suggested by Matsunou et al. [15] to be due to a form of retro-differentiation towards fetal intestine as clear cell carcinoma with morphology similar to the developing gut epithelium, or fetal hepatocytes, i.e., hepatoid carcinoma. AFP-producing colorectal cancers are generally observed to be poorly to moderately differentiated, a histological characterization that makes it different from AFP-producing gastric cancers which are commonly poorly differentiated. However, both rapidly progresses and metastasizes frequently to the liver with a poor prognosis [4]. Poor differentiation, deep submucosal invasion, lymphatic invasion, vascular invasion, or a positive resection margin are risk factors for lymph node metastasis in submucosal colorectal cancers [16].

Due to limited data on CAED, only very few studies and case reports have documented the results of ancillary studies including genomic $[1,13]$ and microsatellite instability (MSI) characteristics [1]. One study reports an MSI-high rate in CAED of $12.2 \%$ [1], which is almost similar to that reported in conventional CRC [17]. The tumor in this case showed intact expression of MMR proteins (MLH-1, PMS-2, MSH-2, MSH-6) by IHC in CAED. NGS analyses from one study revealed that the most frequently mutated gene in CAED is TP53, at a rate of $52.4 \%$ [1] which is almost similar to the TP53 mutation rate in conventional CRC $[18,19]$. The KRAS mutation from the same study was reported at $27.6 \%$, similar to the rate in conventional CRC [20]. We detected a KRAS mutation in our CAED case; however, PD-L1 was negative. Other mutated genes that have been reported in CAED 
include APC (44.8\%), BRAF (20.7\%), NRAS (17.2\%), and PIK3CA (17.2\%) [1]. We recognize the limitation of this case as diagnosis relied on biopsy specimens from the colonic and liver tumors. However, given the advanced stage at disease presentation, neoadjuvant treatment modalities were pursued for possible disease control before surgery.

There are no specific treatment guidelines yet for patients with AFP-producing colorectal cancers. Therefore, treatment regimens are employed according to multiple guidelines and personal experience. Most will undergo the conventional treatments for colorectal cancers which include surgery and chemotherapy with many dying within a year of initiating therapy [21]. Our patient is being evaluated for autologous stem cell transplant following disease progression on standard chemotherapy for conventional CRC and germ cell tumor.

In summary, $\mathrm{CAED}$ is a rare subtype of $\mathrm{CRC}$ with poor prognosis and poses diagnostic difficulties on account of its clinical and histopathologic similarities to primary HCC and germ cell tumors. Ancillary diagnostic modalities including gene expression profiling in the tumor cells by DNA sequencing techniques, may be necessary to correctly diagnose this entity, especially in challenging cases as was encountered in this case. Therefore, pathologists need to be aware of this rare tumor, as a missed diagnosis could significantly impact the clinical care and outcome of patients.

\section{Ethics Statement}

As this is a case study without identifiers, our institution does not require approval from the institutional review board or its equivalent.

\section{Availability of Data and Material}

Data sharing not applicable to this article as no datasets were generated or analyzed during the study.

\section{Code Availability}

Not applicable.

\section{ORCID}

Evi Abada https://orcid.org/0000-0001-5061-2687

Ifeoma C. Anaya https://orcid.org/0000-0001-9941-6660

Anthony Lebbos https://orcid.org/0000-0003-4048-4217

\section{Author Contributions}

Conceptualization: EA, RB. Data curation: EA, RB. Methodology: all authors. Project administration: EA. Resources: all authors. Supervision: all authors. Visualization: all authors. Writing-original draft: all authors. Writing-review \& editing: all authors. Approval of final manuscript: all authors.

\section{Conflicts of Interest}

The authors declare that they have no potential conflicts of interest.

\section{Funding Statement}

No funding to declare.

\section{References}

1. Yamashiro Y, Saito T, Hayashi T, et al. Molecular and clinicopathological features of colorectal adenocarcinoma with enteroblastic differentiation. Histopathology 2020; 77: 492-502.

2. Murakami T, Yao T, Mitomi H, et al. Clinicopathologic and immunohistochemical characteristics of gastric adenocarcinoma with enteroblastic differentiation: a study of 29 cases. Gastric Cancer 2016; 19: 498-507.

3. Liu X, Sheng W, Wang Y. An analysis of clinicopathological features and prognosis by comparing hepatoid adenocarcinoma of the stomach with AFP-producing gastric cancer. J Surg Oncol 2012; 106: 299-303.

4. Anzai H, Kazama S, Kiyomatsu T, et al. Alpha-fetoprotein-producing early rectal carcinoma: a rare case report and review. World J Surg Oncol 2015; 13: 180.

5. Ren F, Weng W, Zhang Q, et al. Clinicopathological features and prognosis of AFP-producing colorectal cancer: a single-center analysis of 20 cases. Cancer Manag Res 2019; 11: 4557-67.

6. Bergstrand CG, Czar B. Demonstration of a new protein fraction in serum from the human fetus. Scand J Clin Lab Invest 1956; 8: 174.

7. O'Conor GT, Tatarinov YS, Abelev GI, Uriel J. A collaborative study for the evaluation of a serologic test for primary liver cancer. Cancer 1970; 25: 1091-8.

8. Bae JM, Lee TH, Cho NY, Kim TY, Kang GH. Loss of CDX2 expression is associated with poor prognosis in colorectal cancer patients. World J Gastroenterol 2015; 21: 1457-67.

9. McIntire KR, Waldmann TA, Moertel CG, Go VL. Serum alphafetoprotein in patients with neoplasms of the gastrointestinal tract. Cancer Res 1975; 35: 991-6.

10. Nakajima T, Okazaki N, Morinaga S, Tsumuraya M, Shimosato Y, Saiki S. A case of alpha-fetoprotein-producing rectal carcinoma. Jpn J Clin Oncol 1985; 15: 679-85.

11. Furuya Y, Wakahara T, Akimoto H, et al. Clear cell adenocarcinoma with enteroblastic differentiation of the ascending colon. J Clin Oncol 2011; 29: e647-9.

12. Murakami T, Yao T, Yatagai N, et al. Colorectal adenocarcinoma with enteroblastic differentiation: a clinicopathological study of five cases. Histopathology 2020; 76: 325-32.

13. Ogiwara S, Furihata M, Fukami K, Yamashita A, Yao T, Osada T. Hepatoid adenocarcinoma with enteroblastic differentiation in the sigmoid colon: lessons from a rare case. Am J Gastroenterol 2019; 114: 684-5.

14. Eloy C, Lopes JM, Faria G, et al. Clear cell change in colonic polyps. Int J Surg Pathol 2009; 17: 438-43.

15. Matsunou H, Konishi F, Jalal RE, Yamamichi N, Mukawa A. Alphafetoprotein-producing gastric carcinoma with enteroblastic differentiation. Cancer 1994; 73: 534-40.

16. Yasuda K, Inomata M, Shiromizu A, Shiraishi N, Higashi H, Kitano S. Risk factors for occult lymph node metastasis of colorectal cancer invading the submucosa and indications for endoscopic mucosal resection. Dis Colon Rectum 2007; 50: 1370-6.

17. Nojadeh JN, Behrouz Sharif S, Sakhinia E. Microsatellite instability in colorectal cancer. EXCLI J 2018; 17: 159-68.

18. Iacopetta B. TP53 mutation in colorectal cancer. Hum Mutat 2003; 21: $271-6$. 
19. Li XL, Zhou J, Chen ZR, Chng WJ. P53 mutations in colorectal cancer: molecular pathogenesis and pharmacological reactivation. World J Gastroenterol 2015; 21: 84-93.

20. Natsume S, Yamaguchi T, Takao M, et al. Clinicopathological and molecular differences between right-sided and left-sided colorectal cancer in Japanese patients. Jpn J Clin Oncol 2018; 48: 609-18.

21. Nakamura Y, Matsuda K, Yokoyama S, et al. Alpha-fetoprotein-producing rectal cancer successfully responded to preoperative chemoradiotherapy: case report. Surg Case Rep 2018; 4: 111. 